\title{
POLÍTICA DE SAÚDE PARA A EPIDEMIA DA AIDS DO BRASIL E MÉXICO NO CONTEXTO DO ACORDO TRIPS ${ }^{(*)}$
}

\author{
HEALTH POLITICS FOR HIV/AIDS EPIDEMY OF BRAZIL \\ AND MÉXICO IN THE CONTEXT OF TRIPS AGREEMENT
}

\author{
Julio Cesar Acosta-Navarro ${ }^{(*)}$ \\ Maria Cristina Cacciamali $i^{(* *)}$ \\ Amaury P. Gremaud ${ }^{(* * *)}$ \\ José R. Carvalheiro ${ }^{(* * * * *)}$ \\ Moacyr C. Nobre ${ }^{(* * * * *)}$ \\ Silvia Cárdenas-Prado ${ }^{(* * * * * *)}$
}

\begin{abstract}
Resumo: Durante as últimas duas décadas, a sociedade testemunhou dois fenômenos sociais que a afetaram substancialmente: a epidemia de HIV/AIDS com suas características pouco usuais e alta letalidade; e a intrusão de leis de propriedade intelectual na vida cotidiana, como o Acordo TRIPS com amplas repercussões sobre a saúde pública. O objetivo deste trabalho foi analisar retrospectivamente como foi a política de saúde de combate à epidemia do HIV/AIDS de Brasil e México. Comparativamente, o impacto da epidemia do HIV sobre a população foi maior no Brasil que no México, gerando no primeiro uma maior preocupação da sociedade civil, levando a classe política a tomar medidas inovadoras e
\end{abstract}

(*) Baseado na tese apresentada ao Programa de Pós-graduação de Integração da América Latina (PROLAM) da Universidade de São Paulo (USP), como requisito para obtenção do título de Doutor em Integração da América Latina (2011): Acordo TRIPS e suas repercussões sobre o acesso a medicamentos. O caso do HIV/AIDS no Brasil e no México: "direito de patente" vs. "direito à vida". Nenhum conflito de interesses. O estudo recebeu apoio financeiro parcial do PROLAM para viagem a cidade de México.

(**) Senior cardiologist, Instituto do Coração (InCor), Faculdade de Medicina da Universidade de São Paulo (FMUSP). E-mail: <jnavarro_2@hotmail.com>.

(***) Professora da Faculdade de Economia, Administração e Contabilidade da Universidade de São Paulo e do PROLAM. E-mail: <cciamali@uol.com.br>.

$(* * * *)$ Professor da Faculdade de Economia, Administração e Contabilidade da Universidade de São Paulo-Ribeirão Preto. E-mail: <agremaud@usp.br>.

$(* * * * *)$ Fundação Oswaldo Cruz. E-mail: <jcarval@fiocruz.com>.

$(* * * * * *)$ Instituto do Coração (InCor), Faculdade de Medicina da Universidade de São Paulo (FMUSP). E-mail: $<\underline{\text { mrnobre@ }}$ usp.br>.

$(* * * * * *)$ Instituto de Psiquiatria, Faculdade de Medicina da Universidade de São Paulo (FMUSP) E-mail: <silcar15@ hotmail.com> Recebido em 02.02.2013 e aceito em 22.05.2013. 
corajosas para enfrentar a epidemia, como aquela do acesso universal ao tratamento drogas antirretrovirais (ARV). Esta política, associada à fabricação de genéricos e uma melhor condição de negociação com empresas farmacêuticas por menores preços de novas drogas ARV mediante disposição de executar licença compulsória de ARVs, garantiram ao Brasil um maior benefício em termos de bem-estar da população.

Palavras chave: Acesso a medicamentos ARVs; Acordo TRIPS; AIDS (Síndrome de imunodeficiência adquirida); Propriedade intelectual; Saúde pública.

\begin{abstract}
Over the last two decades, the society accompaniedtwo social phenomena that affected it greatly: the HIV/AIDS epidemic with its unusual characteristics and high lethality; and the intrusion of intellectual property laws in everyday life, such as the TRIPS Agreement with seriousconsequences on public health. The goal of this work was to analyze retrospectively how health policy was to combat HIV/AIDS epidemic in Brazil and Mexico. Comparatively, the impact of the HIV epidemic on the population was higher in Brazil than in Mexico, generating in the first greater concern of civil society, leading to political class to take courageous and innovative measures to address the epidemic, such as that of universal access to antiretroviral (ARV) treatment drugs. This policy, coupled with the manufacturing of generics and better condition for negotiating with drug companies for lower drug prices through ARV provision performing compulsory license of ARVs guaranteed to Brazil a greater benefit in terms of well-being of the population.
\end{abstract}

Key words: Access to ARVs drugs; AIDS (Acquired Immunodeficiency Syndrome); Intellectual property; Public health; TRIPS Agreement.

\title{
INTRODUÇÃO
}

Durante as últimas duas décadas, a sociedade testemenhou dois fenômenos sociais que deixaram uma profunda reflexão para esta e as próximas gerações. Em primeiro lugar, a epidemia de HIV/AIDS (Vírus da Imunodeficiência Humana/Síndrome de Imunodeficiência Adquirida), com suas caraterísticas históricas pouco usuais e alta letalidade; em segundo lugar, a intrusão de leis de propriedade intelectual na vida cotidiana, como o Acordo TRIPS — (Trade Related Intellectual Property Subjects), com amplas repercusões sobre a saúde pública.

Segundo vários analistas, a epidemia de HIV/AIDS foi de tal magnitude que somente pode ser comparada a dois eventos trágicos na história da humanidade. Primeiro, a epidemia da peste negra, no século XIV (que causou, aproximadamente entre 20 e 30 milhões de mortes, quase 2/3 da população europeia da época) (MAYER, 2006), e depois, a epidemia da influenza (gripe) espanhola, no início do século XX (estimada em 50 milhões de mortes) (MORENS, 2008). Em países desenvolvidos, a introdução de tratamento antirretroviral (ARV) altamente ativo e a disponibilidade de drogas para as infeções oportunistas levaram a uma redução substancial na mortalidade pela AIDS. Diversos fatores inter-relacionados determinam o acesso às drogas essenciais, incluindo aquelas para tratar o HIV e as infecções oportunistas. Entre eles, estão: uso apropriado, aspectos econômicos, seleção da droga, legislação e regulação, fabricação, pesquisa e decisões de desenvolvimento (COHEN-KHOLER, 2008). Como a epidemia de HIV/AIDS é bem recente na história médica, a maioria das drogas criadas especialmente para tratar a infecção pelo HIV e mudar o curso evolutivo fatal da AIDS já está sob patentes. Isto torna o tratamento contra a AIDS menos acessível em comparação àqueles para os quais existem alternativas de medicamentos genéricos. 
A proteção de patentes, neste caso, garante direitos exclusivos para uma invenção e evita a concorrência de genéricos. Esta certamente é uma das razões possíveis para a limitada disponibilidade de medicamentos e a dificuldade no cumprimento do tratamento. Neste contexto, muitos estudiosos, consideram que o Acordo TRIPS seja responsável pela crise global atual de medicamentos para o HIV/AIDS (SATYANARAYANA, 2005).

O objetivo deste trabalho foi analisar retrospectivamente o significado da interseção destes dois fenômenos nos últimos anos nos casos do Brasil e do México. Pretendemos responder à seguinte questão: Como foi a política de saúde de combate à epidemia do HIV do Brasil e do México — em termos do modelo do sistema de saúde, atendimento aos portadores de HIV, acesso a medicamentos e resultados-no contexto do Acordo TRIPS? Os procedimentos metodológicos foram baseados na análise de fontes primárias de relatos e opiniões de profissionais de diversas instituições envolvidas na problemática em ambos os países e no exame de fontes secundárias (documentos oficiais, livros, jornais, publicações especializadas e sites oficiais de diversas instituições).

\section{RESULTADOS E DISCUSÃO}

\section{PANORAMA HISTÓRICO DO TRATAMENTO DA EPIDEMIA DO HIV/AIDS}

Sem tratamento com medicação ARV, o curso fatal da AIDS leva aproximadamente um ano (KALLINGS, 2008). No início da epidemia, o tratamento era focado sobre as infecções oportunistas. Levou algum tempo para se achar um tratamento melhor. O tratamento com medicamentos ARV se iniciou em 1987 com zidovudina (AZT). Curiosamente, AZT é um produto resultante da colaboração sistemática entre uma empresa privada (British WelcomeTrust) e outra pública (National Cancer Institute-NCI, dos Estados Unidos). Ambas as instituições tinham o AZT na suas reservas de componentes com possível atividade citotóxica para tratamento do câncer, e acreditou-se que o AZT, sendo um análogo de nucleósido (timidina), poderia inibir a síntese de HIV. Houve remarcável sucesso inicial, mas o tratamento com AZT como monoterapia não teve sucesso em razão do desenvolvimento de resistência viral e um número elevado de efeitos colaterais. Nos anos 90, outros análogos de nucleósidos foram desenvolvidos, também como os inibidores de transcriptasa reversa não nucleósidos. Drogas com outros modos de ação foram introduzidas, incluindo inibidores da protease e inibidores da entrada, e hoje já há mais de vinte diferentes drogas no mercado. Porém, a verdadeira revolução veio com o tratamento que combinava três diferentes drogas, introduzido em 1996, desse modo, reduzindo o risco de resistência e incrementando a eficácia no longo prazo. Em 6 de fevererio de 2001, a companhia indiana Cipla ofereceu terapia tripla para tratamento de AIDS por 350 dólares por paciente/ano à ONG Médicos sem Fronteiras (MSF) e a 600 dólares por paciente/ano para governos de países em desenvolvimento. Naquela época, o preço do mesmo "coquetel" de laboratórios transnacionais estava entre 10 e 15 mil dólares por paciente/ano. A dramática redução de preços da Cipla, que recebeu destaque na mídia, deixou claro o fato de que as companhais multinacionais estavam abusando de sua posição monopolista em meio a um desatre humano catastrófico (HOEN, 2009). 


\section{O IMPACTO DA EPIDEMIA DE HIV NO BRASIL E NO MÉXICO}

A América Latina é a terceira região, depois da África e da Ásia, em comprometimento de pessoas infectadas pelo HIV. Na América Latina, o total estimado de novas infecções pelo HIV em 2008 foi de 170 mil e, em consequência, o número de pessoas que vivem com o vírus ascende a 2 milhões. Os dados mais recentes sugerem que a epidemia se mantém estável na América Latina. A prevalência regional do HIV é de 0,6\%, caracterizando a epidemia na região como de baixo nível e concentrada (UNAIDS/WHO, 2009). O Brasil e o México são os maiores países da América Latina em área territorial, os mais populosos e as maiores economias da região. Os dados epidemiológicos sobre HIV/AIDS no Brasil e no México são apresentados na Tabela 1. No Brasil, a AIDS tem se configurado como epidemia concentrada. No início da década de 1980, ela atingiu principalmente os usuários de drogas injectáveis (UDI), homossexuais e bissexuais masculinos (HSH), assim como os indivíduos que receberam transfusão de sangue e hemoderivados. O primeiro caso de AIDS foi notificado retrospectivamente na cidade de São Paulo em 1980. A este caso inicial seguiram-se outros, basicamente restritos às denominadas metrópoles nacionais, tendo como categorias de exposição preponderantes os HSH, os hemofílicos e as demais pessoas que receberam sangue e hemoderivados. Em razão de os hemofílicos receberem habitualmente componentes sanguíneos de um conjunto (pool) de doadores - até então, habitualmente pagos pelas doações — , este segmento populacional foi rápida e profundamente atingido no início da epidemia no Brasil. Entretanto, à extensa disseminação inicial seguiu-se certa estabilização em anos posteriores, em especial entre aqueles homens pertencentes às camadas médias urbanas, em meio aos quais verificou-se relevante mobilização social e mudanças de comportamento no sentido de práticas sexuais mais seguras (SZWARCWALD, 2000).

Até meados da década de 1990, os coeficientes de mortalidade eram crescentes. Hoje, o índice se mantém estável, com cerca de 11 mil óbitos anuais desde 1998 e uma taxa de 6 por 100 mil habitantes (Tabela 1); até 2008, calcula-se em mais de 200 mil óbitos em decorrência do HIV/AIDS. Após a introdução da política de acesso universal ao tratamento ARV, a mortalidade caiu e a sobrevida aumentou (BRASIL, 2009). Em média, cerca de 30 mil novos casos de AIDS são identificados a cada ano, com números crescentes entre mulheres e pessoas com mais de 50 anos.

O México, da mesma forma que o Brasil, também é classificado como um país em que a epidemia de AIDS é concentrada, difundindo-se rapidamente em um ou mais subgrupos da população. O primeiro caso de AIDS no México foi registrado em 1983. Segundo as estimativas realizadas pelo CENSIDA, de maneira conjunta com a UNAIDS, no momento, existem no país 220 mil adultos infectados pelo HIV (MÉXICO, CENSIDA, 2009). No México, antes da introdução do tratamento ARV, a taxa de mortalidade geral em razão da AIDS era de 1,8 mortes entre cada 100 mil habitantes (1990). Em 1996, atingiu seu máximo registro com 4,8 mortes. A partir daquele ano, o progressivo acesso a um novo grupo de ARVs constituindo o TARV, mudou radicalmente o prognóstico dos pacientes com AIDS e melhorou sustancialmente sua qualidade de vida. Por este motivo, de 1998 em diante, a mortalidade geral por AIDS se manteve entre 4,2 e 4,4 mortes em cadal00 mil habitantes. Análises efetuadas posteriormente identificaram que a sobrevida média dos pacientes 
com AIDS anterior a 1996 era de 17 meses; mas, a partir daquele ano, se estendeu para 57 meses graças ao TARV (RODRIGUES, 2008). Embora tenha sido alcançado o acesso universal ao tratamento ARV, ainda não se percebe o impacto desta importante medida de saúde pública nos dados de mortalidade. No ano 2000, a taxa de mortalidade por SIDA entre a população geral foi de 4,3 mortes entre cada 100 mil habitantes, enquanto que em 2007 foi de 4,8. As taxas de mortalidade do grupo de 25-44 anos de idade mostraram, no entanto, uma leve tendência decrescente, que indica que o período de sobrevida das pessoas infectadas tem se dilatado (MÉXICO, CENSIDA, 2009).

\section{O SISTEMA DE ASSISTÊNCIA À EPIDEMIA DE HIV NO BRASIL E NO MÉXICO}

Indicadores do sistema de atenção à epidemia de HIV no Brasil e no México são monstrados na Tabela 2. A atual Constituição da República Federativa do Brasil, de 1988, dispõe que a saúde é direito de todos e dever do Estado, garantido mediante políticas sociais e econômicas que visem à redução do risco de doença e de outros agravos e pelo acesso universal igualitário às ações e serviços para sua promoção, proteção e recuperação. Os princípios básicos do SUS, estruturado a partir de 1990, são a universalidade, a equidade e a integralidade (BRASIL, 2010).

O caso do México, diferentemente do Brasil, foi um típico exemplo de um sistema de saúde fragmentado. Diferentes instituições atuam de forma independente nas funções de financiamento e provisão de serviços e possuem sua própria rede de cuidados básicos, assim como hospitais de segundo e terceiro nível ("integração vertical") (IZAZOLA, 2000). Os recursos públicos financiam a operação de dois tipos básicos de instituições públicas de saúde: as instituições de seguridade social — Instituto Mexicano del Seguro Social (IMSS), Instituto de Seguridad y ServiciosSociales de los Trabajadores del Estado (ISSSTE), Petróleos Mexicanos (PEMEX), Secretaría de la Defensa (SEDENA) e Secretaría de Marina (SEMAR) — e as instituições dedicadas a atender à população sem seguro social - Secretaría de Salud e IMSS - Oportunidades (IMSS-O). Em 2000, cerca de 40\% da população careciam de seguridade social, sendo que o IMSS dava cobertura a $47 \%$ da população geral (BAUTISTA, 2003).

Posteriormente, em maio de 2003, se publica o decreto pelo qual se reforma a Ley General de Salud para criar o Sistema de Protección Social en Salud (SPSS). Esta estratégia garante o acesso integral aos serviços públicos de saúde para todos os mexicanos, sob um sistema de seguridade universal em saúde, sem distinção de sua condição social, laboral e econômica, proporcionando suporte financeiro às pessoas sem acesso às instituições de seguridade social (TOUMESH, 2008).

Dentro deste contexto, podemos datar o início da resposta brasileira à AIDS em 1983, quando o governador Franco Montoro e o secretário da saúde responderam às reivindicações da comunidade e ao movimento sanitarista com a organização do programa de combate à AIDS em São Paulo. Desde o início o Programa de AIDS de São Paulo, foi organizado com todos os componentes que existem até hoje no programa, começando pela prevenção, passando pela vigilância epidemiológica, assistência, o reconhecimento de direitos humanos e um forte componente de articulação com organizações da sociedade 
civil (TEIXEIRA, 2003). Atualmente, o Programa Nacional de DST e AIDS, do Ministério da Saúde, criado em 1986, representa a autoridade nacional no enfrentamento da epidemia.

No México, o combate à epidemia do HIV se iniciou em fevereiro de 1986, quando foi criado o CONASIDA (Consejo Nacional para la Prevención y Control del SIDA), organizado em vários subcomitês que ficaram responsáveis pelas tarefas de prevenção e controle da infecção, tais como educação para a saúde, pesquisa, vigilância epidemiológica e bancos de sangue.

\section{POLÍTICA DE ACESSO O TRATAMENTO ARV}

No caso do Brasil, em 1991, o governo brasileiro, atendendo a reivindicações de setores da sociedade civil, iniciou a distribuição da AZT para milhares de pessoas vivendo com HIV/AIDS com indicação de tratamento no país. A iniciativa de entrega de medicamentos à população causou preocupação nos meios científicos internacionais, pelo risco aventado da possibilidade de disseminação de formas resistentes de HIV, plausabilidade biológica que eventualmente poderia resultar de tratamentos incompletos, interrupção precoce do tratamento ou de prescrição inadequada decorrentes de uma insuficiência no fornecimento das drogas ARV pelo estado brasileiro devido a seu alto custo. A partir de 1996, em consonância com a legislação nacional que ampara a distribuição gratuita e universal dos medicamentos, foram incorporados ao elenco de drogas disponibilizadas pelo Ministério da Saúde os ARVs potentes, como os inibidores de protease e de fusão. Em 2007, dezoito ARVs compunham o conjunto de drogas disponibilizadas ao tratamento de pessoas com HIV no Brasil, sendo oito deles produzidos no país por seis diferentes laboratórios estatais e um laboratório privado, em 37 formulações (pediátricas e de adultos) (BRASIL, 2010).

No México, de 1983 a 1996, os cuidados aos enfermos de AIDS se concentraram em hospitais de especialidades; porém, até 1992, o tratamento dos pacientes se limitava a apoio psicológico em paralelo ao tratamento das infecções oportunistas e neoplasias. A partir de 1991, o Ministerio de Salud começou a destinar ARVs para um número, embora muito limitado, de pessoas pobres, sob protocolos de pesquisa. Ante esta situação, surgiram organizações de pessoas infectadas que começaram a demandar o acesso a medicamentos e uma atenção integral e de qualidade. Surge a "Frente de Personas Afectadas por el VIH/AIDS" (FRENPAVIH), que fortaleceu a mobilização social já existente e fez novas demandas, que acabaram provocando medidas governamentais (MAGIS, 2009).

Em fins de 1997, a disponibilidade da maior parte destes medicamentos unicamente nas instituições de seguridade social chamou atenção para a profunda desigualdade nas opções de atendimento para as pessoas com HIV/AIDS que não tinham acesso a estas instituições. No ano de 1998, o ministro da Saúde mexicano assumiu o compromisso político de proprocionar acesso universal aos ARVs, mas o governo federal não alocou recursos suficientes para isso, transferindo somente 9 milhões de dólares para o Fondo Nacional del SIDA (FONSIDA), ONG criada especificamente para financiar o acesso aos ARVs. Estes fármacos tinham sido primeiramente prescritos por médicos privados para uma minoria da população rica do país e depois pelos médicos de instituições da seguridade social, as primeiras a fornecer acesso público à ARVs. Em 1998, também foi conquistada a cobertura de ARVs para menores de 18 anos e mulheres grávidas, um contingente de infectados sem acesso à seguridade social. Nas palavras da Dra. Uribe, tinha 
se iniciado lentamente o acesso aos ARVs por meio de doações, mas obviamente isto era insuficiente. "El gobierno federal lograba transferir con economías y asignaciones especificas recursos a este fondo, que estaba incialmente a cargo de la Facultad de Medicina da UNAM. Lo llamaron 'El Titanic', porque al principio con los recursos disponibles no se alcanzó a cubrir el tratamiento de todos los pacientes, solo de mujeres y niños” (MÉDICOS DE MÉXICO 2008). Em 1999, a cobertura de ARV se estendeu a mais de 1 mil pacientes sem acesso à seguridade social. Na elaboração do Programa Nacional de Salud 1997-2001, foi criado o "primer modelo para la atención médica para paciente con VIH/SIDA", conhecido como Servicios Especializados para la atención del SIDA (SEAs), que consistia na integração de uma equipe de saúde multidisciplinar com o objetivo de proporcionar atenção ambulatorial e integral nos hospitais. Durante o ano de 2001, o CONASIDA se transformou no Centro Nacional para la Prevención y Control del SIDA (CENSIDA), funcionando como um organismo autônomo da Secretaria de Salud. No período 2001-2006, o México adota a política de acesso universal ao atendimento médico integral em HIV, que inclui o tratamento ARV para todas as pessoas com HIV sem seguro social. O programa, inciado em 2001, estendeu a cobertura a todas as pessoas sem seguridade social somente em 2004. Em concordância com o "Programa Nacional de Salud 2007-2012" e o programa de ação "Respuesta al VIH/SIDA e ITS 2007-2012" - que tem como um de seus objetivos melhorar o acesso e a qualidade da assistência integral a todas as pessoas com HIV e outras infecções de transmisão sexual (ITS) —, o Governo Federal definiu o acesso universal a medicamentos ARVs como um dos eixos prioritários para diminuir os estragos causados pelo vírus HIV. Pelo "Fideicomiso de Gastos Catastróficos del Sistema de Protección Social en Salud", a Secretaría de Salud, atualmente, garante a compra destes medicamentos para distribuição gratuita a todas as pessoas com HIV que não tenham seguridade social, tanto para quem já recebe tratamento como para os novos casos que se incorporem. Cerca de 27\% das 220 mil pessoas que vivem com HIV recebem terapia ARV em alguma das instituições de saúde do país ou na medicina privada (MÉXICO, CENSIDA, 2009).

\section{Custos}

Indicadores de financiamento e distribuição de custos dos sistemas de atenção à epidemia de HIV/AIDS são apresentados na Tabela 3. No caso do Brasil, estima-se que mais de $80 \%$ do orçamento do Ministério da Saúde para compra de ARVs sejam utilizados para a aquisição de onze medicamentos patenteados e $20 \%$ para a compra de sete medicamentos fabricados por laboratórios nacionais. Esta enorme proporção do orçamento gasto com medicamentos patenteados tem colocado em risco a sustentabilidade e a universalidade deste programa de atenção à saúde. Esta ameaça à sustentabilidade do programa levou o governo brasileiro a travar uma verdadeira disputa de patentes pelo mecanismo da "licença compulsória" (contemplada no artigo 68 da sua Lei de Propriedade Industrial) com laboratórios transnacionais e o governo dos Estados Unidos. Diversos estudos demonstram que há uma drástica redução no preço dos medicamentos tão logo as patentes dos produtos de referência expiram, em virtude da concorrência de produtos genéricos que passam a ficar disponíveis no mercado. No que se refere ao tratamento da AIDS, graças à concorrência dos medicamentos genéricos, o preço da terapia tríplice de primeira linha 
(estavudina, lamivudina e nevirapina) caiu de 10.439 dólares por paciente/ano em 2000 (menor preço do original), para 87 dólares porpaciente/ano (menor preço internacional - genérico Hetero) em 2008, ou seja, uma redução de mais de 99\% no preço do tratamento, possível pelo fato de estes medicamentos não estarem protegidos por patentes em determinados países, o que permitiu aos laboratórios farmacêuticos locais a produção de versões genéricas (VIEIRA, 2009).

A manutenção da sustentabilidade da política nacional do Brasil encontrou importante suporte no fato de o governo federal ter concedido a licença compulsória do medicamento efavirenz, no primeiro semestre de 2007, que permitiu reduzir o valor do medicamento em $72,2 \%$ (BRASIL, 2008). No Brasil, um estudo realizado no final de 2007 demonstrou uma economia de recursos públicos de mais de 1 bilhão de dólares, de 2001 a 2005, apenas no que se refere à compra de 6 medicamentos ARVs, graças à negociação de preços em um cenário internacional de concorrência em função da existência de medicamentos genéricos (NUNN, 2007). Diferentes estudos demonstram que, em um cenário de concorrência, o preço dos medicamentos reduz-se drasticamente e, consequentemente, aumenta o número de pessoas com acesso a tratamento que pode salvar suas vidas (VIEIRA, 2009).

No caso do México, o gasto total com HIV/AIDS no período compreendido entre 1997 e 2009 aumentou 307\% (valores corrigidos pela inflação). No âmbito da prevenção, o aumento foi gradativo (110\%), em contraste com o incremento exponencial que, até 2008, se observa na área da assistência, relacionada com o acesso a tratamento ARV que, no final de 2006, se converteu efetivamente num programa universal no país (591\%). Uma característica importante na questão do acesso a medicamentos ARVs no México, diferente em relação ao debate brasileiro, foi a maior preocupação da sociedade civil mexicana; por exemplo, com a qualidade dos medicamentos. Parte dos argumentos enfatizava que, para ter esta garantia, estes medicamentos teriam que ser comprados exclusivamente dos laboratórios detentores das patentes, o que dificultou a popularização de medidas como licenças compulsórias ou importações paralelas. Porém, a este raciocínio deve ser contraposto o fato de que a OMS tem um programa de pré-qualificação de medicamentos que conduz as atividades de avaliação e inspeção e contribui para a construção de capacidades nacionais para a fabricação de medicamentos de alta qualidade, incluindo ARVs. A OMS começou a avaliar medicamentos ARV em 2001. Atualmente, a lista de produtos medicinais pré-qualificados inclui 178 formulações de ARV, das quais 56 são originárias de companhias inovadoras versus 122 de empresas genéricas. É nossa impressão que o raciocínio de considerar como única alternativa para o acesso a medicamentos ARVs de alta qualidade as negociações com laboratórios transnacionais detentores de patentes serviu de justificativa, tanto ao governo quanto à sociedade civil, para evitar discutir temas mais espinhosos como licença compulsória ou importação paralela. Tudo isto claramente no contexto da situação econômica política do México em relação aos Estados Unidos.

Devemos reconhecer que dentro das limitações da nossa pesquisa uma foi a incompletude de dados em alguns indicadores ou dados para fazer uma melhor análise comparativa vis-à-vis; e que outra, foi a dificuldade metodológica natural para tratar uma problemática essencialmente multidisciplinar, a mesma que tem aspectos de índole de saúde pública, economia, relações internacionais e direito entre outras ciências. Recentemente publicamos 
uma complementação desta pesquisa tratando aspectos de legislação de propriedade intelectual entre estes dois países e uma contextualização geral e mais ampla da problemática (ACOSTA-NAVARRO, 2012).

\section{CONCLUSÕES}

Apesar da dificuldade metodológica natural para tratar uma problemática essencialmente multidisciplinar, como um programa específico de saúde que é determinado por múltiplos fatores e atores como Estado, sociedade civil, comunidade científica, organizações internacionais e empressa privada, focamos principalmente sobre a política de saúde para a epidemia da AIDS de ambos os países. Embora Brasil e México tenham caraterísticas demográficas relativamente semelhantes, tenham tido seu primeiro caso de AIDS reportado no mesmo ano, assim como iniciaram programas específicos de combate à epidemia do HIV cronologicamente contemporâneos, seguiram por caminhos diferentes, principalmente quanto à decisão de acesso universal ao tratamento ARV e suas respectivas consequências. A política de acesso universal implantada precocemente, a fabricação de genéricos e uma melhor condição de negociação com empresas farmacêuticas por menores preços de novas drogas ARV pela disposição de executar licença compulsória de ARVs - com o objetivo de garantir a sustentabilidade do programa de combate à epidemia garantiram ao Brasil um maior benefício em termos de bem-estar da população afetada pela epidemia.

\section{REFERÊNCIAS BIBLIOGRÁFICAS}

ACOSTA-NAVARRO, Julio. Direito de patentes vs. direito à vida. A epidemia da AIDS no contexto do Acordo TRIPS. Curitiba, Juruá, 2012.

BAUTISTA, Sergio; DMYTRACZENSKO, Tania; KOMBE, Gilbert; BERTOZZI, Stegano. Costing of HIV/AIDS treatment in Mexico. Technical Report n. 20, Bethesda, MD. Partners for health Reform Plus, 2003.

BRASIL. Ministério da Saúde. DST/AIDS. Boletim Epidemiológico AIDS-DST, versão 2009.

MINISTÉRIO DA SAÚDE. Metas e compromissos assumidos pelos Estados-membros na sessão especial da Assembleia Geral das Nações Unidas sobre HIV/AIDS. UNGASS - HIV/AIDS. Resposta Brasileira 2008-2009 — Relatório de Progresso do País. Brasília, 2010.

Ministério da Saúde do Brasil/Secretaria de Vigilância em Saúde/Programa Nacional de DST e AIDS. Metas e compromissos assumidos pelos Estados-membros na sessão especial da Assembleia Geral das Nações Unidas em HIV/AIDS. UNGASS - HIV/AIDS. Resposta Brasileira 2005/2007 — Relatório de Progresso do País — Programa Nacional de DST. Brasil, fevereiro 2008.

COHEN-KHOLER, Jillian Clare; FORMAN, Lisa \& LIPKUS, Nathaniel. Addressing legal and political barriers to global pharmaceutical access: options for remedying the impact of the Agreement on Trade-Related Aspects of Intellectual Property Rights (TRIPS) and the imposition of TRIP-plus standards. Health Economics, Policy and Law, 2008.

HOEN, Ellen. The global politics of pharmaceutical monopoly power. AMB publishers: Holanda, 2009.

IZAZOLA, José Antonio (editor). Cuentas nacionales en VIH/SIDA. Estimación de flujos de financiamiento y gasto en VIH/SIDA. Fundación Mexicana para la Salud. México, 2000. 
KALLINGS, L. O. The first postmodern pandemic: 25 years of HIV/AIDS. Journal of Internal Medicine, 2008

MAGIS, Carlos; PARRINI, Rodrigo. Nuestra epidemia. El SIDA en México 1983-2002. In: MAGIS, Carlos; BARRIENTOS, Hermelinda. VIH/SIDA y salud pública. CENSIDA 2009.

MAYER, Kenneth; PIZER, H. F; VENKATESH, Kartik. The social ecology of HIV/AIDS. Medicine Clinics of North America, 2008.

MÉDICOS DE MÉXICO. 25 años de VIH en México, ano VI, n. 87, CONASIDA - CENSIDA: Una época de cambios significativos. Edic. Agosto 2008.

MÉXICO, CENSIDA (Centro Nacional para la Prevención y Control del SIDA). El VIH/SIDA em México 2009. Disponível em: <www.censida.gob.mx>.

MORENS, David; FOLKERS, Gregory; FAUCI, Anthony. Emerging infections: a perpetual challenge. The Lancet Infection, 2008.

NUNN, Amy; FONSECA, Elize; BASTOS, Francisco; GRUSKIN, Sofia; SALOMON, Joshua. Evolution of antiretroviral drugs costs in Brazil in the context of free and universal access to AIDS treatment. PLOS Medicine; 4 (11), November 2007. Disponível em: <http://www.plosmedicine. org/article/info:doi/10.1371/journal.pmed.0040305>.

RODRIGUEZ, Carlos, HERNANDEZ, Mauricio. Epidemiología del SIDA en México. In: VILLALOBOS, José; PONCE DE LEÓN, Samuel; VALDESPINO, José. 25 años de SIDA en México: logros, desaciertos y retos. México, 2008. Disponível em: <www.censida.gob.mx>.

SATYANARAYANA, K. TRIPS, patents \& HIV/AIDS drugs. Indian Journal of Medical Research, 2005.

SZWARCWALD, Célia Landmann; BASTOS, Francisco; ESTEVES, Maria; ANDRADE, Carla. A disseminação da epidemia da AIDS no Brasil, no período de 1987-1996: uma análise espacial. Cad. Saúde Pública, Rio de Janeiro, 16 (Sup. 1): 7-19, 2000.

TEIXEIRA, Paulo. Acesso universal a medicamentos para AIDS: a experiência do Brasil. Divulgação em Saúde em Debate. Rio de Janeiro, agosto 2003.

TOUMEH, Daniel. EL sistema de protección social en salud y El VIH/SIDA. In: VILLALOBOS, José, PONCE DE LEÓN, Samuel; VALDESPINO, José. 25 años de SIDA en México. Logros, desaciertos e retos. Instituto Nacional de Salud Pública. México, 2008.

UNAIDS. Epidemiological Fact Sheeton HIV and AIDS. Brazil E Mexico. Dezembro 2008. Disponível em: <www.unaids.org >

UNAIDS-08. 2008 Report on the global AIDS epidemic. Disponível em: <www.unaids.org>.

UNAIDS. Scaling up priority HIV/AIDS interventions in the health sector 2008. Progress report, p. 10, 2008.

UNAIDS/WHO. Situação da epidemia de AIDS 2009 - Dados sobre a América Latina, 2009. Disponível em: <www.unaids.org.br> Acesso em: 10 abr. 10.

VIEIRA Marcelo; MACHADO Eloisa (Org). Acesso a medicamentos: audiência pública sobre saúde. Rio de Janeiro: ABIA, 2009. 


\section{APÊNDICES}

Tabela 1 - Indicadores epidemiológicos da epidemia de HIV/AIDS no Brasil e no México*

\begin{tabular}{|c|c|c|}
\hline Estimativas e dados epidemiológicos & Brasil & México \\
\hline $\begin{array}{l}\text { Extensão territorial }\left(\mathrm{km}^{2}\right) \\
\text { População - } 2009 \\
\text { Ranking por número de pessoas infectadas pelo HIV na } \\
\text { América Latina }\end{array}$ & $\begin{array}{r}8.514 .876 \\
192.811 .000 \\
1^{\underline{o}}\end{array}$ & $\begin{array}{r}1.958 .201 \\
109.610 .000 \\
2^{\underline{0}}\end{array}$ \\
\hline $\begin{array}{l}\text { Estimativa de pessoas infectadas pelo HIV - Adultos + } \\
\text { crianças (menores de } 14 \text { anos) - } 2008\end{array}$ & 730.000 & 220.000 \\
\hline $\begin{array}{l}\text { Estimativa de pessoas infectadas pelo HIV - Adultos } \\
\text { (maiores de } 15 \text { anos) - } 2008\end{array}$ & $\begin{array}{l}710.000 \\
(97,3 \%)\end{array}$ & $\begin{array}{c}210.000 \\
(96 \%)\end{array}$ \\
\hline $\begin{array}{l}\text { Estimativa de crianças com HIV } \\
(0-14 \text { anos })\end{array}$ & $\begin{array}{l}20.000 \\
(2,7 \%)\end{array}$ & $\begin{array}{c}10.000 \\
(4 \%)\end{array}$ \\
\hline Casos notificados de AIDS até $2009^{* *}$ & $\begin{array}{l}544.846 \\
\text { H: } 67 \% \\
\text { M: } 23 \%\end{array}$ & $\begin{array}{l}135,003 \\
\text { H: } 82,3 \% \\
\text { M: } 17,7 \%\end{array}$ \\
\hline Prevalência de infecção por HIV em adultos (15-49 anos) & $0,6 \%$ & $0,3 \%$ \\
\hline Taxa de mortalidade (por 100 mil habitantes) & 6,1 & 4,8 \\
\hline Óbitos por AIDS somente em 2007 & 15.000 & 11.000 \\
\hline Óbitos até 2008 & 217.091 & 72.000 \\
\hline
\end{tabular}

* Fontes: BRASIL, Boletim Epidemiológico AIDS-DST, versão, 2009; México, CENSIDA 2009. El VIH/ SIDA en México 2009.

UNAIDS. Epidemiological Fact Sheet on HIV and AIDS. Brazil E México. Dezembro 2008. Disponível em: <www.unaids.org>.

** No caso do Brasil, a porcentagem é em função do total de casos de homens (H) ou de mulheres (M). No caso do México, a porcentagem é do total de casos de ambos sexos.

Tabela 2 - Indicadores do sistema de atenção à epidemia de HIV no Brasil e no México

\begin{tabular}{|l|c|c|}
\hline \multicolumn{1}{|c|}{$\begin{array}{c}\text { Indicadores do sistema de atenção } \\
\text { à epidemia de HIV }\end{array}$} & Brasil & México \\
\hline Primeiro caso registrado de AIDS & $\begin{array}{c}1980 \text { (somente } \\
\text { identificado em } \\
1982)\end{array}$ & 1983 \\
\hline Início do acesso a medicamentos ARVs & 1991 & 1997 \\
\hline Início do “Acesso universal” aos ARVs & 1996 & 2001 \\
\hline $\begin{array}{l}\text { Estimativa do número pessoas recebendo terapia } \\
\text { ARV - 2007 }\end{array}$ & 181.000 & 43.000 \\
\hline $\begin{array}{l}\text { Estimativa do número de pessoas } \\
\text { precisando da terapia ARV - 2007 }\end{array}$ & 230.000 & 76.000 \\
\hline
\end{tabular}




\begin{tabular}{|l|c|c|}
\hline Cobertura da terapia ARV (\%) - 2004 e 2007 & $\begin{array}{c}74(2004) \\
80(2007)\end{array}$ & $\begin{array}{c}45(2004) \\
57(2007)\end{array}$ \\
\hline Crianças menores de 15 anos recebendo terapia ARV & $6.815(2007)$ & $176(2006)$ \\
\hline Indicadores de prevenção da transmissão mãe-filho & & 146 \\
\hline $\begin{array}{l}\text { Número de grávidas com HIV que receberam terapia } \\
\text { ARV para prevenção de transmissão ao filho - 2007 }\end{array}$ & 6.188 & 3.100 \\
\hline $\begin{array}{l}\text { Número de grávidas com HIV precisando de ARVs } \\
\text { para prevenção - 2007 }\end{array}$ & 8.600 & 5 \\
\hline $\begin{array}{l}\text { Porcentagem de grávidas com HIV que receberam } \\
\text { ARVs para prevenção - 2007 }\end{array}$ & 72 & 5 \\
\hline
\end{tabular}

Fonte: UNAIDS, Epidemiological Fact Sheet on HIV and AIDS. Brazil \& México. December 2008. Disponível em: <www.unaids.org>.

\section{Tabela 3 - Indicadores de custos do sistema de atenção à epidemia de HIV/AIDS no Brasil e no México*}

\begin{tabular}{|l|c|c|}
\hline & Brasil (2006) & México (2005) \\
\hline Gasto nacional em saúde/PIB - 2007 & $6,7 \%$ & $5,1 \%$ \\
\hline $\begin{array}{l}\text { Gasto total - Domésticos públicos e internacionais } \\
\text { (milhões de dólares) }\end{array}$ & 565.186 & 176.052 \\
\hline Gastos domésticos públicos (\% do total) & $99,5 \%$ & $99,2 \%$ \\
\hline Gastoscom recursos de fonte internacional: & $0,1 \%$ & $0,1 \%$ \\
Bilateral & $0,0 \%$ & $0,0 \%$ \\
Fundo global & $0,3 \%$ & $0,0 \%$ \\
Nações Unidas e outras agências & $0,0 \%$ & $0,7 \%$ \\
Outras fontes internacionais & 34,159 & 40,663 \\
\hline Gasto total em prevenção (milhões de dólares) & 470,055 & 122,333 \\
\hline Gasto total em cuidados e tratamento (milhões de & & 103.396 \\
dólares) & 438.384 & 0,036 \\
\hline Terapia ARV (milhões de dólares) & 0,148 & \\
\hline Gastos com órfãos e crianças vulneráveis (milhões & & \\
de dólares) & & \\
\hline
\end{tabular}

Fontes: UNAIDS, Epidemiological Fact Sheet on HIV and AIDS. Brazil \& México. Dezembro 2008. Disponível em: <www.unaids.org>.

BRASIL. Ministério da Saúde do Brasil/Secretaria de Vigilância em Saúde/Programa Nacional de DST e AIDS. Metas e compromissos assumidos pelos Estados-membros na sessão especial da Assembleia Geral das Nações Unidas em HIV/AIDS. UNGASS - HIV/AIDS. Resposta Brasileira 2005/2007 - Relatório de Progresso do País - Programa Nacional de DST. Brasil, fevereiro 2008.

UNAIDS-08. 2008 Report on the global AIDS epidemic. Disponível em: <www.unaids.org>. 